\title{
Review
}

\section{Intracellular lectins are involved in quality control of glycoproteins}

\author{
By Kazuo Yamamoto*1,†
}

(Communicated by Kunihiko SuzUKI, M.J.A.)

\begin{abstract}
Glycoprotein quality control is categorized into three kinds of reactions; the folding of nascent glycoproteins, ER-associated degradation of misfolded or unassembled glycoproteins, and transport and sorting of correctly folded glycoproteins. In all three processes, $\mathrm{N}$-glycans on the glycoproteins are used as tags that are recognized by intracellular lectins. We analyzed the functions of these intracellular lectins and their sugar-binding specificities. The results clearly showed that the A, B, and C-arms of high mannose-type glycans participate in the folding, transport and sorting, and degradation, respectively, of newly synthesized peptides. After correctly folded glycoproteins are transported to the Golgi apparatus, $N$-glycans are trimmed into $\mathrm{Man}_{3} \mathrm{GlcNAc}_{2}$ and then rebuilt into various complex-type glycans in the Golgi, resulting in the addition of diverse sugar structures that allow glycoproteins to play various roles outside of the cells.
\end{abstract}

Keywords: lectin, specificity, quality control, folding, transport, ER-associated degradation

\section{Biochemical aspects of sugar-recognition}

Glycosylation of proteins occurs in the endoplasmic reticulum and the Golgi apparatus and is the most sophisticated post-translational modification of proteins. Glycosylation is a highly abundant form of post-translational modification, and glycosylated proteins are estimated to comprise approximately $70-80 \%$ of all proteins in the cell. ${ }^{1)}$ Like other posttranslational modifications, glycan-mediated interactions play an important role in numerous cellrecognition processes, such as trafficking and degradation of proteins and cells in blood, as well as during immune responses, differentiation of cells, cell-cell adhesion, and cell-matrix interactions. ${ }^{1)}$ Since many

*1 Department of Integrated Biosciences, Graduate School of Frontier Sciences, The University of Tokyo, Chiba, Japan.

$\dagger$ Corresponding should be addressed: K. Yamamoto, Graduate School of Frontier Sciences, The University of Tokyo, Kashiwa, Chiba 277-8562, Japan (e-mail: yamamoto@k.u-tokyo. ac.jp).

Abbreviations: CNX: calnexin; ER: endoplasmic reticulum; ERAD: ER-associated degradation; ERGIC: ER-Golgi intermediate compartment; ERGIC-53: ER-Golgi intermediate compartment protein of $53 \mathrm{kDa}$; ERp57: endoplasmic reticulum $57 \mathrm{kDa}$ protein; EDEM: ER-degradation enhancing mannosidaselike protein; FAC: frontal affinity chromatography; $K_{a}$ : association constant; MPR: mannose 6-phosphate receptor; VIP36: vesicular integral membrane protein of $36 \mathrm{kDa}$; VIPL: VIP36-like protein; OS-9: osteosarcoma protein 9. biological phenomena are regulated by molecular recognition, research techniques that provide accurate information on the specific interactions that occur between molecules are essential for a better understanding of biological events. Several methods have been developed that can predict strong interactions between molecules and allow us to achieve a better understanding of many intercellular and intracellular phenomena. The interaction between immunoglobulin and antigen has high affinity $\left(K_{a}=10^{7}-10^{11} \mathrm{M}^{-1}\right)$ and they form stable complex. Since rapid switching on and off are essential for cellular responses under rapidly changing environmental conditions, the association between intracellular signaling molecules, such as transcription factors, kinases or phosphatases associated with transmembrane receptors and adaptor proteins involved in transducing signals, are occasionally regulated in their binding to cognate proteins by their phosphorylation/dephosphorylation cycles, or abrogated by degradation via ubiquitination. ${ }^{2)}$ In contrast to the strong interactions that occur between these molecules, weak interactions $\left(K_{a} \sim\right.$ $10^{4} \mathrm{M}^{-1}$ ), such as those between sugars and sugarbinding proteins, especially animal lectins, are also involved in a dynamic range of cellular activities. ${ }^{3)}$ In the case of cellular and molecular recognition mediated via sugar-protein interactions, the self- 
association of both receptors and their ligands on cell surfaces causes an increase in avidity between receptors and ligands by one to three orders of magnitude, which is required for signaling into the cell. $^{4), 5)}$ Similar to self-association mechanisms such as the clustering or patching of interacting biomolecules, the dissociation of each molecule from the formed complex occurs without modification of these molecules and results in the switching off of the cellular signal. ${ }^{6)}$ Though several immunoassays with high sensitivity, including precipitation, agglutination, enzyme-linked immunosorbent assay (ELISA), and flow cytometry, are widely used, such methodologies fail to detect weak interactions; thus, insight into the role of sugar-protein interactions in physiological processes is limited using such conventional methods.

To address these difficulties with regard to the study of sugar-binding proteins, we established two highly sensitive methods using lectin tetramers and cell-surface display of lectins to investigate the sugarbinding specificities and other significant characteristics of lectins. Using these techniques, we analyzed all of the putative intracellular lectins present in the endoplasmic reticulum (ER) and Golgi apparatus. Furthermore, based on these data, functional analyses of these intracellular lectins with respect to quality control of glycoproteins in the ER and the Golgi apparatus of a cell were performed using cellular and molecular biological, biochemical, and structural approaches.

\section{Novel methodologies for monitoring weak sugar-protein interactions}

Animal lectins act as receptors for sugarcontaining ligands. Identification of the ligands recognized by each receptor is requisite for understanding their biological functions, such as sugarmediated signaling. However, animal lectins are quite different from classical plant lectins in their sugarbinding ability; the $K_{a}$ values of plant lectinsugar interactions range from approximately $10^{6}$ to $10^{7} \mathrm{M}^{-1}$, while those of animal lectin-sugars are much weaker, approximately $10^{4} \mathrm{M}^{-1}$.4),5) Furthermore, animal lectins cannot be easily detected without specific antibodies because they are expressed at very low levels. We have established a highly sensitive method for monitoring the binding of animal lectins to sugars, lectin tetramer-binding to membranebased glycans, ${ }^{7)}$ and reporter assay using cell-surface display. ${ }^{8)}$ Multimerization of lectins and sugar ligands is a rational and facile approach for in- creasing the avidity of their interaction to detectable thresholds; however, regulation of multimerization is essential, especially for a comparative analysis of wild-type and mutated lectins. Firstly, we established an approach to prepare lectin tetramers and assess their binding to cell-surface carbohydrates by flow cytometry. We developed methods that allowed us to 1) prepare soluble lectins fused to a biotinylation sequence followed by enzymatic biotinylation, 2) obtain $R$-phycoerythrin-labeled lectin tetramers, and 3) measure tetramer binding to various lectinresistant cell lines or cells treated with sugarprocessing inhibitors. Our second approach used methods that allowed us to 1) establish cells expressing chimeric lectin/CD3 $\zeta$ fusion proteins in BWZ.36 reporter cells, 2) prepare a 96-well culture plate containing immobilized sugar polymers, lectinresistant $\mathrm{CHO}$ cells, or $\mathrm{CHO}$ cells treated with several sugar-processing inhibitors, 3) culture the lectin/CD3 $\zeta$ fusion protein-expressing reporter cells, and 4) measure the induced $\beta$-galactosidase activity by colorimetric assay. The lectin-displaying reporter cells express a chimeric protein of the lectin domain/ CD3 $\zeta$ fusion protein on their cell surfaces, and crosslinking of lectins on the cell surface induces $\beta$ galactosidase expression in the cell (Fig. 1). This method is especially favourable for the analysis of intracellular lectins. These two molecular and cellular biochemical approaches, along with frontal affinity chromatography (FAC), ${ }^{9)}$ offers several advantages over existing approaches and allows us to detect the weak sugar-binding activity of lectins $\left(K_{a} \sim\right.$ $10^{4} \mathrm{M}^{-1}$ ), especially animal lectins, and elucidate their specific sugar ligands. Because intracellular lectins localize in organelles, it is difficult to directly measure their sugar-binding activity, so this cellsurface-display approach offers distinct advantages.

\section{The glycan processing pathways of glycoproteins in the ER and the Golgi}

Most proteins are posttranslationally modified with phosphate, sulfate, acetate, lipids, or carbohydrates, and such modifications play important roles in their functions. Among these modifications, glycosylation is the most complicated process, since glycan complexity in combination with the similar physico-chemical properties of glycans allows glycans to accomplish a wide variety of cellular functions. ${ }^{10)}$ Glycoprotein function is dependent on sugar structures, as this mediates recognition by sugar binding proteins, lectins. Lectins can recognize and distinguish between various sugar sequences and struc- 
A a soluble lectin domain with a biotinylation tag

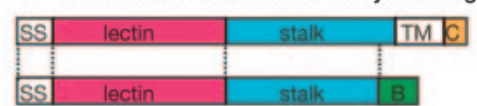

a membrane-bound chimeric lectin

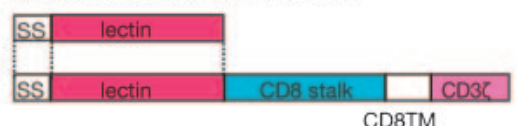

B
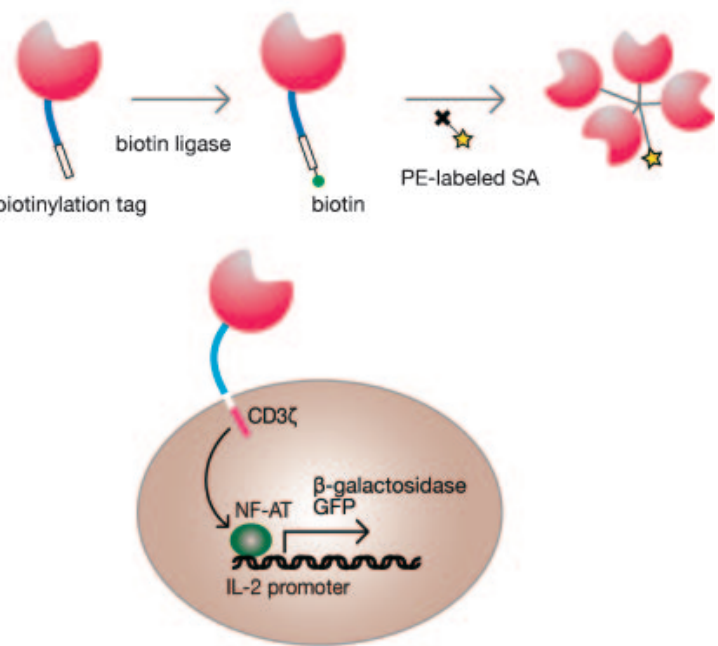

Fig. 1. (A) Preparation of plasmids encoding a soluble lectin domain with a biotinylation tag and a membrane-bound chimeric lectin. SS indicates signal sequence; Lectin, lectin domain; stalk, stalk domain; TM, transmembrane domain; C cytoplasmic domain; B, biotinylation sequence; CD8 stalk, CD8 stalk domain; CD8TM, CD8 transmembrane domain; CD3 $\zeta$, CD3 $\zeta$ cytoplasmic domain. (B) Scheme of the production of a soluble lectin tetramer for use in flow cytometry. PE-labeled SE indicates $R$-phycoerythrin-labeled streptavidin. (C) Activation of reporter cells through chimeric lectins displayed on the cell surface is monitored by expression of $\beta$-galactosidase or green fluorescent protein (GFP). NF-AT indicates nuclear factor of activated T-cells; IL-2, interleukin-2.

tures, therefore facilitating the initiation of complex biological processes by sugar-recognition. ${ }^{3)}$

There may be several aspects to be taken into account when considering the complicated roles played by the sugar chains of glycoproteins. One is the classification of sugar-recognition processes based on diverse or general roles among biological species. One of the roles of sugar chains that is conserved among species is quality control of glycoproteins. Figure 2 shows the biosynthetic pathway of typical $\mathrm{N}$-glycans and $\mathrm{O}$-glycans on glycoproteins. It is well known that the glycosylation process occurs in both the ER and the Golgi apparatus of the cell. However, the glycosylation processes of $\mathrm{N}$-glycans and $O$ glycans are quite different. ${ }^{11)}$ Firstly, $N$-glycosylation occurs just after the translation of glycoproteins in the ER, whereas $O$-glycosylation does not occur in the ER. Secondly, $N$-glycan precursors are synthesized on dolichol pyrophosphate as 14 mers $\left(\mathrm{Glc}_{3} \mathrm{Man}_{9} \mathrm{GlcNAc}_{2}\right)$ consisting of glucose, mannose, and $\mathrm{N}$-acetylglucosamine and are then transferred to Asn-X-Ser/Thr ( $\mathrm{X}$ is any amino acid except Pro) sequences on nascent polypeptides by oligosaccharyltransferase (OST). In contrast, $O$-glycans are sequentially elongated one by one. Thirdly, $N$ glycans are trimmed in the ER and then sequentially elongated in the Golgi. By contrast, $O$-glycans are gradually synthesized to full length. Interestingly, the biosynthetic pathway of $\mathrm{N}$-glycans is conserved in a wide variety of organisms, including yeast, insects, plants, and animals. By contrast, $O$-glycans are produced via a number of synthetic pathways and adopt a variety of structures in various species. More than 30 years after the processing pathways of $N$ and $O$-glycans were revealed, glycobiologists are still unable to explain why such an exhaustive and complicated pathway is used for $\mathrm{N}$-glycan synthesis compared to that of $O$-glycan.

\section{Quality control of glycoproteins is mediated by intracellular lectins}

Recently, we comprehensively analyzed all of the intracellular proteins with lectin domains. The results demonstrated that $\mathrm{N}$-glycans in the ER serve as tags for quality control of glycoproteins. Quality control of glycoproteins includes three kinds of reactions, namely, the folding of newly synthesized peptides into a proper structural conformation, the degradation of misfolded proteins or unassociated subunits by the proteasome in the cytosol, and the sorting of proteins during their transport from the ER to the Golgi and between the cisternae of the Golgi apparatus. ${ }^{12), 13)}$ In each cell, nearly 5,000 proteins are synthesized; approximately $70 \%$ of these are folded correctly, ${ }^{14), 15)}$ whereas the remainder of the proteins are unable to function correctly due to a folding error. Because these misfolded proteins can cause damage and abrogate cell function, they are translocated into the cytosol and destined for degradation by the proteasome. The functional proteins are sorted into the proper transport pathways during their movement from the ER through the Golgi. These three mechanisms of protein processing, folding, and ER-associated degradation and sorting, are essential for cell viability. These processes are rigorously checked and regulated by intracellular lectins in the ER and the Golgi, with various $\mathrm{N}$-glycan structures acting as tags. It is 


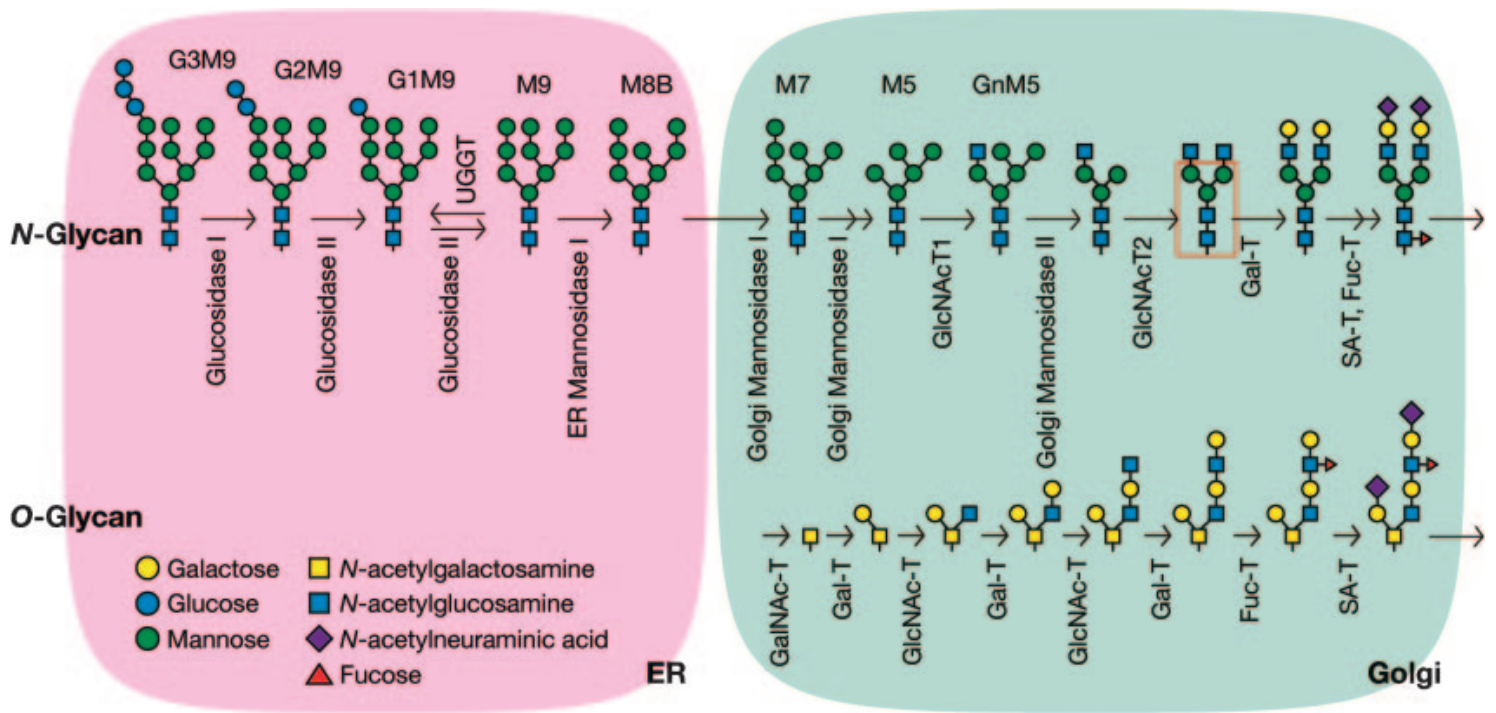

Fig. 2. Processing of oligosaccharides is distinctly different in the ER and the Golgi. $N$-glycan processing occurred in the ER are conserved in eukaryotes, and the glycans function as markers for quality control of glycoproteins. By contrast, $N$ - and $O$-glycans synthesized and processed in the Golgi exhibit a wide variety of structures consisting of several kinds of sugar residues.

A

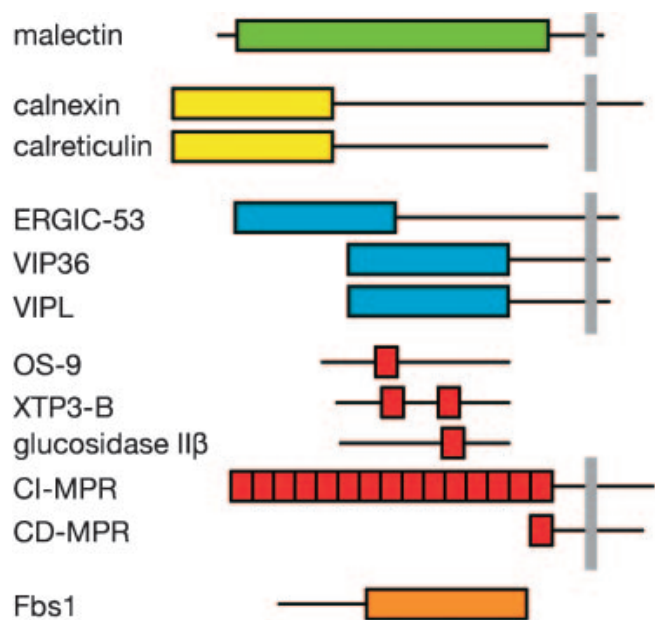

B

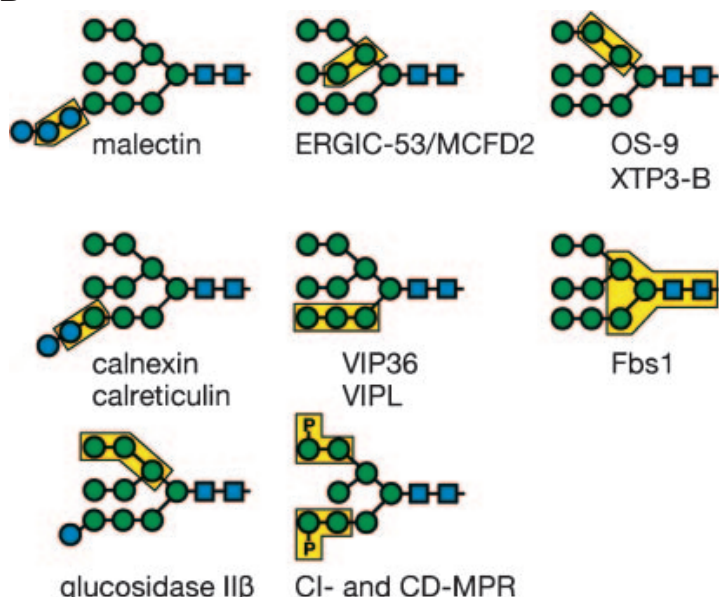

Fig. 3. Schematic illustration of intracellular lectins involved in quality control of glycoproteins and their sugar-binding specificities.

(A) Schematic illustration of intracellular lectins involved in the quality control process. Boxes indicate their lectin domains.

(B) Structures of $N$-glycans that serves as ligands for intracellular lectins. Sugar residues essential for tight binding are indicated by yellow.

remarkable that lectins, which are relatively small proteins, are able to regulate quality control of much larger proteins by recognizing the sugar moieties attached to these peptides (Fig. 3).

4.1. The folding of newly synthesized proteins is assisted by malectin, calnexin and glucosidase II $\beta$. During translation in the ER, proteins are simultaneously glycosylated with $N$-glycan precursors by OST on asparagines in an Asn-X-Ser/ Thr sequence before the peptide undergoes folding.
The transferred $N$-glycan precursor consists of three glucose, nine mannose, and two $N$-acetylglucosamine residues. ${ }^{11)}$ For newly translated peptides to be folded correctly in the ER, they must be modified with these $\mathrm{N}$-glycans precursors.

The $N$-glycan precursor, $\mathrm{Glc}_{3} \mathrm{Man}_{9} \mathrm{GlcNAc}_{2}$ (G3M9), is not bound by any of the ER-resident lectins. However, when the outermost $\alpha 1,2$-linked glucose residue is removed by type II transmembrane glucosidase I (GI), it becomes a ligand for a 


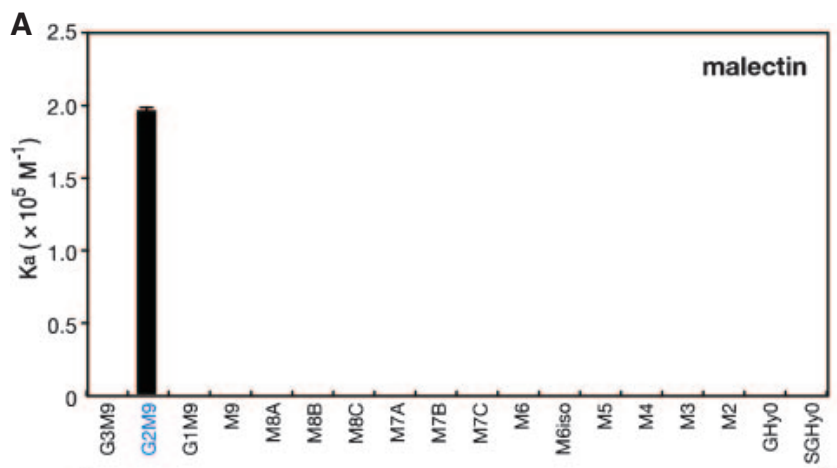

B
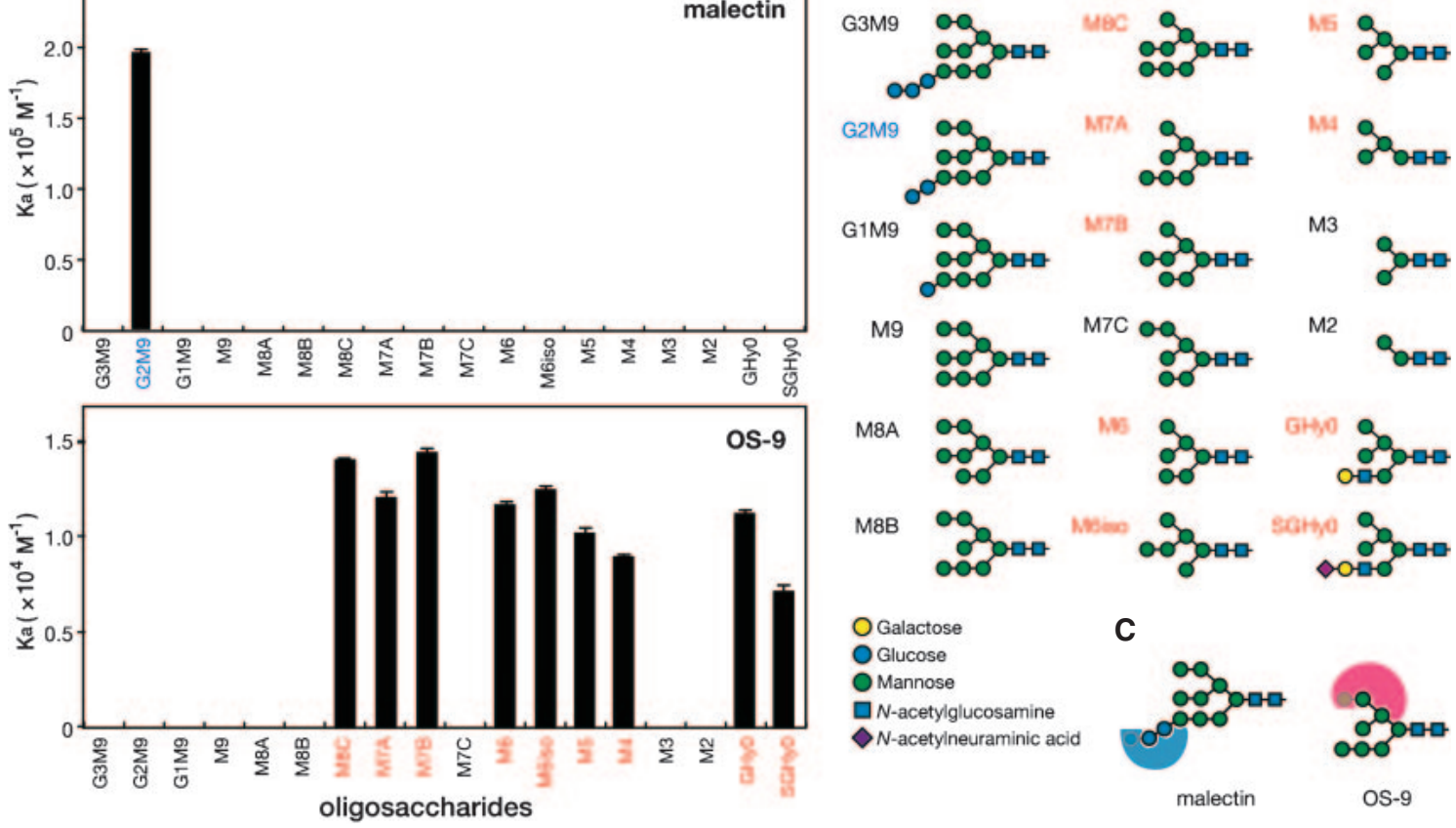

M3

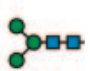

M2
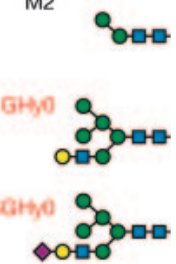

malectin

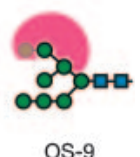

Fig. 4. (A) The affinity of each 2-aminopyridine (PA)-labeled $N$-glycans for human malectin and OS-9 were measured by frontal affinity chromatography and $K_{a}$ values are shown. Modified from Refs. 16 and 34. (B) Structures of $N$-glycans used in FAC analysis. (C) Model for malectin and OS-9 recognition of oligosaccharide ligands.

novel membrane-anchored ER lectin called malectin. Malectin was first identified in Xenopus laevis, and is highly conserved in animals. Using a membranebased lectin tetramer binding assay (Fig. 1B) and $\mathrm{FAC},{ }^{16)}$ we demonstrated that human malectin specifically recognizes $\mathrm{Glc}_{2} \mathrm{Man}_{9} \mathrm{GlcNAc}_{2}$ (G2M9) with a $K_{a}$ of $1.97 \times 10^{5} \mathrm{M}^{-1}$, but binds neither $\mathrm{Glc}_{1} \mathrm{Man}_{9} \mathrm{GlcNAc}_{2}$ (G1M9) nor G3M9 (Fig. 4). We further demonstrated that like other molecular chaperones, human malectin is induced under conditions of ER stress and preferentially associates with folding-defective glycoproteins. ${ }^{17)}$ Malectin preferentially associated with a folding-defective $\alpha 1$-antitrypsin variant, null Hong Kong ( $\left.\mathrm{AT}^{\mathrm{NHK}}\right)$, and reduced its secretion; however, it did not affect the secretion of wild-type (WT) $\alpha 1$-antitrypsin (AT) ${ }^{16)}$ Galli et al. also reported that prolonged malectin association with folding-defective and misfolded glycoproteins affected processing of $\mathrm{N}$-glycans and substantially reduced secretion of these cargo proteins. ${ }^{18)}$ To clarify the molecular mechanisms by which malectin preferentially associates with misfolded glycoproteins, we performed a proteomic analysis of proteins that coimmunoprecipitated with malectin and showed that malectin constitutively co-immunoprecipitated with ribophorin I. Ribophorin I is an ER-resident transmembrane protein that serves as a subunit of the mammalian OST complex. ${ }^{19)}$ Recent studies show that ribophorin I regulates the glycosylation of some glycoproteins by selectively associating with, and delivering them to, the catalytic core of the OST complex. ${ }^{20), 21)}$ Interestingly, we demonstrated that ribophorin I preferentially interacts with misfolded ribonuclease A, but not with the native form, suggesting that ribophorin I functions as a chaperone inside the cell. Molecular modeling of the 3D structure of the lumenal domain of ribophorin I demonstrated that ribophorin I has several $\beta$ propeller domains rich in hydrophobic amino acids on its molecular surface. These findings suggest a role for malectin in the quality control of glycoproteins via the formation of a complex with ribophorin I, as illustrated Fig. 5.

When the terminal $\alpha 1,3$-linked glucose residue is removed from G2M9 by glucosidase II (GII), it binds to the ER-resident transmembrane lectin, calnexin (CNX). CNX is the first discovered ER-resident lectin whose precise function was clearly demonstrated. ${ }^{22), 23)}$ In the ER, CNX specifically binds to the G1M9 glycan to trap newly synthesized peptides and 


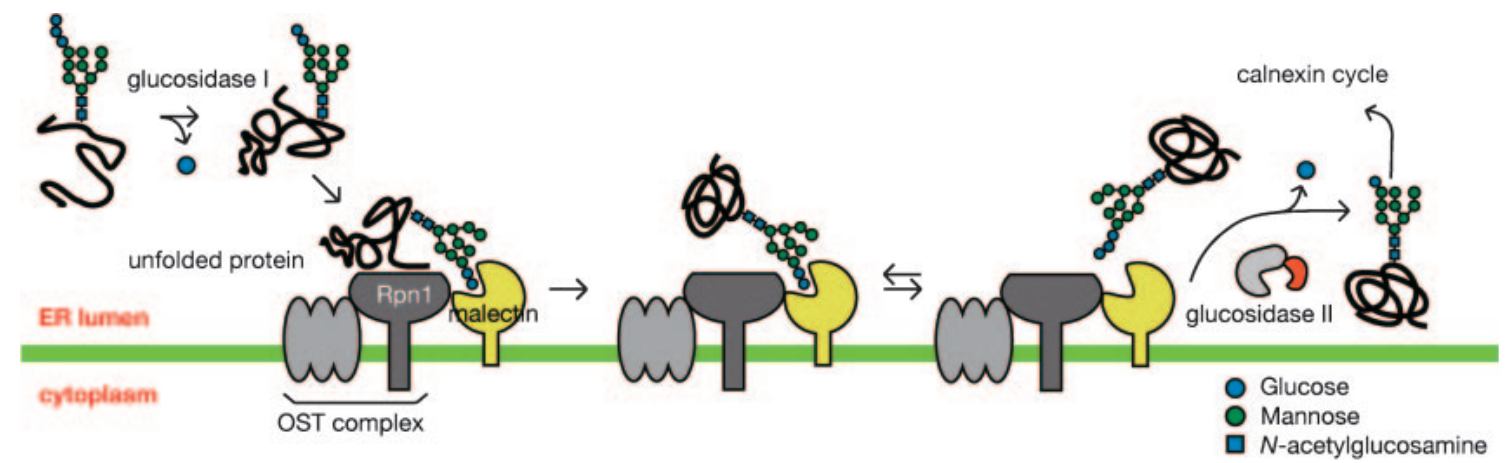

Fig. 5. Role of malectin-ribophorin I complex in quality control of glycoproteins. Malectin forms a complex with ribophorin I (Rpn1), which is one of the subunits of the oligosaccaryltransferase complex (OST complex). Malectin specifically binds to G2M9 glycan, whereas ribophorin I preferentially binds to unfolded or misfolded proteins. Modified from Ref. 17.

initiate folding in conjunction with chaperones, protein disulfide isomerase and peptidyl-prolyl cistrans isomerase. It is known that CNX associates with the disulfide isomerase ERp57, which contributes to the correct folding of unfolded proteins. ${ }^{24)}$ Binding of CNX to the sugar moieties of unfolded proteins facilitates folding of the peptide by CNXassociated chaperones. Cleavage of the final $\alpha 1,3-$ linked glucose by GII results in the dissociation of CNX, allowing the correctly folded glycoprotein to proceed to the Golgi apparatus for further processing to complex- or hybrid-type glycoforms. When the released glycoprotein remains to be correctly folded, UDP-glucose:glycoprotein glucosyltransferase (UGGT) recognizes the hydrophobic patch on the unfolded portion of the glycoprotein or unassociated subunit and reglucosylates the $\mathrm{Man}_{9} \mathrm{GlcNAc}_{2}$ (M9) $N$-glycans of these proteins to G1M9. ${ }^{25)}$ The reglucosylated protein then again binds to CNX, and further folding will proceed. This reaction cycle is called the CNX cycle (Fig. 6).

In contrast to GI, which is a membrane-bound glycosidase, GII is a soluble heterodimeric complex comprised of $\alpha$ and $\beta$ subunits. The GII $\alpha$ subunit $(\mathrm{GII} \alpha)$ contains a catalytic domain belonging to the hydrolase family 31 , while the GII $\beta$ subunit $(\mathrm{GII} \beta)$ possesses a mannose 6 -phosphate receptor homology (MRH) domain, which was first identified as a domain commonly conserved between the calciumdependent-mannose 6-phosphate receptor (MPR) and calcium-independent-MPR. ${ }^{26}$ ) Using a lectin tetramer binding assay and FAC, we examined the sugar-binding ability and specificity of the GII $\beta$ $\mathrm{MRH}$ domain (GII $\beta$-MRH) and identified G1M9, M9, $\mathrm{Man}_{8} \mathrm{GlcNAc}_{2}$ isomers A and B (M8A, M8B) and $\mathrm{Man}_{7} \mathrm{GlcNAc}_{2}$ isomer $\mathrm{C}(\mathrm{M} 7 \mathrm{C})$ as potential

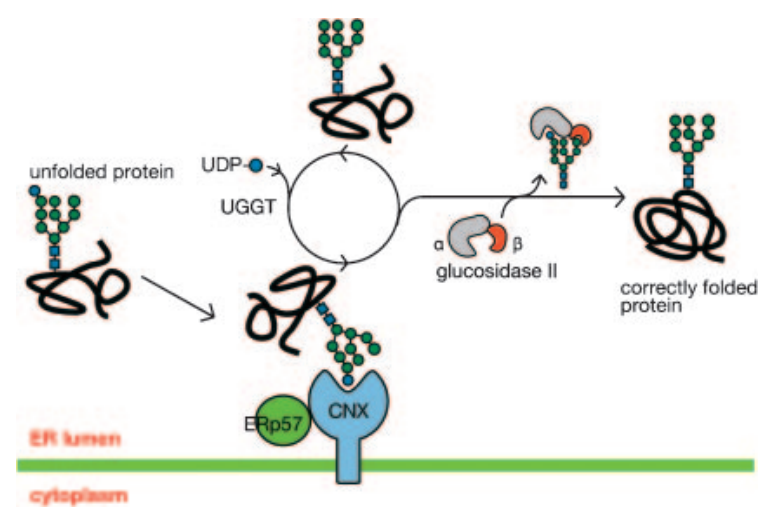

Fig. 6. Calnexin cycle for folding of nascent proteins. Calnexin (CNX) specifically binds to G1M9 glycan on newly synthesized proteins, where ERp57 associated with CNX assists protein folding. If the glycoprotein is not properly folded, UDPglucose:glycoprotein glucosyltransferase (UGGT) will add a glucose residue on the glycan for rebinding to $\mathrm{CNX}$.

ligands of GII $\beta$-MRH. ${ }^{27)}$ Our results suggest that the $\alpha 1,2$-linked mannobiose (Man $\alpha 1,2 \mathrm{Man}$ ) at the nonreducing termini, particularly on the $\mathrm{C}$-arm of high mannose-type glycans, is requisite for GII $\beta-\mathrm{MRH}$ recognition of high mannose-type glycans. It has been reported that in the absence of $\operatorname{GII} \beta, \mathrm{GII} \alpha$ is catalytically inactive, since $\mathrm{GII} \alpha$ is unstable without GII $\beta$. However, the role of the sugar-binding activity of GII remained unknown. Therefore, we examined the role of GII $\beta$-mediated sugar binding in GII enzymatic activity. When the glucosidase activity of GII $\alpha$ associated with either wild-type GII $\beta$ or GII $\beta$ mutants lacking sugar-binding ability was measured, the hydrolysis of a small synthetic substrate, $p$ nitrophenyl- $\alpha$-glucoside $(p \mathrm{NP}-\alpha \mathrm{Glc})$, occurred to a similar extent. In contrast, when native G1M9 or 
G2M9 were used as substrates, GII $\alpha$ associated with mutated GII $\beta$ significantly impaired glucose trimming of both of the native substrates. ${ }^{27)}$ Enhancement of deglucosylation madiated by GII $\beta$ was also reported by another group. ${ }^{28)}$ Deprez et al. reported that removal of the outermost glucose by GI occurs immediately after $\mathrm{N}$-glycosylation to produce the G2M9 glycan. However, efficient trimming of the second glucose from G2M9 by GII only occurs after the second $N$-glycan is transferred to the same polypeptide. ${ }^{29)}$ Based on our data and these observations, we proposed a model in which G2M9binding to the MRH domain of GII $\beta$ induces a conformational change in the catalytic region of GII $\alpha$, thus increasing GII activity and allowing the first cleavage to proceed (Fig. 6, mannose bindinginduced activation). ${ }^{27)}$ This activation of GII may occur efficiently on correctly folded glycoproteins; however, in misfolded glycoproteins, newly transferred $N$-glycan would be folded irregularly into the peptide and not easily accessible to GI and GII, resulting in inefficient trimming of another G2M9. This slow trimming of glucose residues via GI and GII maintains unfolded and misfolded glycoproteins so that they can be associated with malectin and/or CNX.

4.2. ER-associated degradation of misfolded proteins via OS-9 and XTP3-B. After CNX and UGGT are released from the peptides, correctly folded proteins are transported to the Golgi. However, terminally misfolded or unassembled polypeptides within the ER are destined for degradation in the cytoplasm, since these proteins can sometimes cause defects in cellular function. This process is known as ER-associated degradation (ERAD). ${ }^{30}$ ) Misfolded proteins are translocated across the ER membrane, conjugated with ubiquitin, and targeted to the $26 \mathrm{~S}$ proteasome for degradation in the cytoplasm (Fig. 7). To understand the ERAD molecular machinery, Carvalho et al. carried out studies on multi-protein complexes associated with the SEL1L/HRD1 ER ubiquitin ligase in mammals. ${ }^{31)}$ OS-9 and XTP3-B are soluble proteins that associate with HRD1, one of the subunits of the ubiquitin ligase complex. ${ }^{32), 33)}$ Interestingly, OS-9 and XTP3-B have one and two MRH domain(s) in their sequences, respectively. Using a lectin tetramer binding assay and FAC, we demonstrated that $N$-glycans containing terminal $\alpha 1,6$-linked mannose were good ligands for OS-9 and XTP3-B, with $K_{\mathrm{a}}$ values of approximately $1.0 \times 10^{4} \mathrm{M}^{-1}$, and that binding occurred in a cation-independent manner. Trimming of an $\alpha 1,6-$

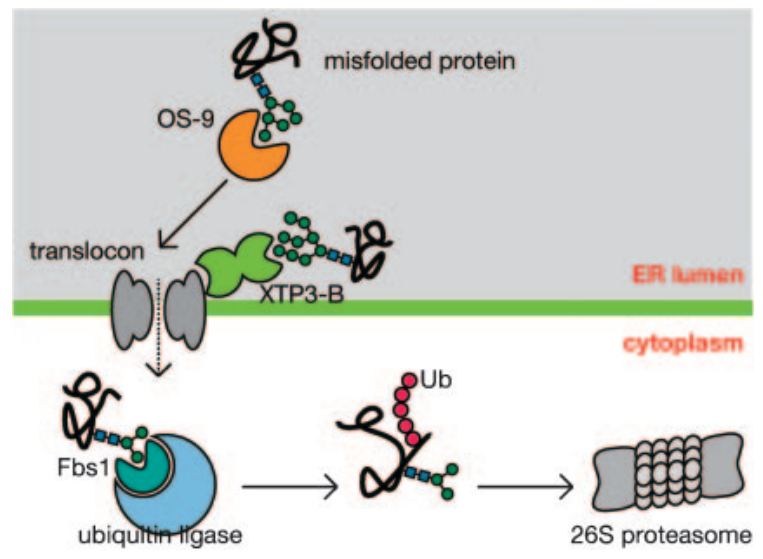

Fig. 7. ER-associated degradation (ERAD) of misfolded proteins. OS-9 and XTP3-B bind to $N$-glycans of ERAD substrates and guide their entry into ERAD. Misfolded proteins are transported to the cytoplasm, ubiquitinated by ubiquitin ligase, and then degraded by proteasome.

linked mannose from the C-arm abrogated the binding of OS-9 and XTP3-B (Fig. 4A). ${ }^{34), 35)}$ Using immunoprecipitation experiments, we demonstrated that the misfolded $\alpha 1$-antitrypsin variant $\mathrm{AT}^{\mathrm{NHK}}$, but not WT $\alpha 1$-antitrypsin, selectively interacted with OS-9 and XTP3-B in the cells. The amount of $\mathrm{AT}^{\mathrm{NHK}}$ that precipitated with sugar-binding deficient OS-9 mutants was significantly lower than that precipitated with WT OS-9, and less OS-9 was precipitated with non-glycosylated $\mathrm{AT}^{\mathrm{NHK}}$ than $\mathrm{AT}^{\mathrm{NHK}}$. These results suggest that trimming of the outermost $\alpha 1,2$-linked mannose on the $\mathrm{C}$-arm is critical for misfolded proteins to enter ERAD. Recently, Hosokawa's group clearly demonstrated that XTP3-B stably associated with ubiquitin ligase complex, HRD1-SEL1L, and that its C-terminal MRH domain recognized M9 glycan, suggesting that XTP3-B may protect nascent immature polypeptides from premature degradation. This indicates that XTP3-B and OS-9 may play important but distinct roles in regulating misfolded/unfolded glycoproteins. $^{36)}$

This observation was also confirmed by X-ray crystallographic analysis of the complex comprised of the OS-9 MRH domain and $\alpha 3$, $\alpha 6$-mannopentaose. The OS-9 MRH domain contains a typical P-type lectin fold, and OS-9 specifically recognizes Man $\alpha 1,6$ Man $\alpha 1,6$ Man on the $\mathrm{C}$-arm via the continuous double tryptophan motif and canonical residues of the MRH domain (Fig. 4C). ${ }^{37)}$ Furthermore, it was demonstrated that these amino acids are involved in the recognition of ERAD substrates such as $\mathrm{AT}^{\mathrm{NHK}}$. 
The MRH domain of OS-9 is critically different from those of calcium-dependent and calcium-independent MPR, which preferentially bind to $\alpha 1,2$-linked mannose 6 -phosphate residues but not to $\alpha 1,6$-linked mannose 6-phosphates. ${ }^{38)}$ Mapping of the hydrophobic residues on the MRH domain of OS-9 reveals no large hydrophobic areas on the molecular surface, suggesting that a putative folding sensor domain exists in the N-terminal and/or C-terminal domain(s), but not in the MRH domain.

What type of glycosidase(s) digests $\alpha 1,2$-linked mannose in the C-arm for ERAD? In mammals, transfection of the gene encoding ER-degradation enhancing mannosidase-like protein (EDEM) 1 or 3 into cells enhanced ERAD of misfolded proteins, suggesting that this protein has $\alpha 1,2$-mannosidase activity specific for the C-arm of high mannose-type glycans. ${ }^{39)}$ These proteins were originally identified as ER stress-induced proteins homologous to ER $\alpha$-mannosidase I; however, they showed no enzymatic activity. ${ }^{40)}$ In yeast, it was currently demonstrated that EDEM orthologue, Htm1p, processed $\mathrm{Man}_{8} \mathrm{GlcNAc}_{2}$ oligosaccharide to $\mathrm{Man}_{7} \mathrm{GlcNAc}_{2}$ in complex with disulfide isomerase Pdi1p. ${ }^{41)}$

4.3. Active transport of cargo proteins from the ER to the Golgi via ERGIC-53. Correctly folded proteins are transported from the ER via the cargo receptor, ER-Golgi intermediate compartment protein 53 (ERGIC-53), a homo-oligomeric ER-Golgi recycling protein. ERGIC-53 is a membrane-bound receptor consisting of an L-type lectin domain, a stalk domain, a transmembrane domain, and a short cytoplasmic domain. ERGIC-53 has both ER-exit and ER-retrieval signals (KKFF motif) in its short cytoplasmic tail. During early secretion, newly synthesized glycoproteins are packaged into COPIIcoated transport vesicles that bud from the rough $\mathrm{ER}$ and move to the ER and Golgi intermediate compartment (ERGIC). ${ }^{42)}$ ERGIC-53 mediates the formation of budding vesicles via the binding of C-terminal double phenylalanine residues (FF) to COPII coat proteins. Similar to the function of a double phenylalanine signal, the dilysine ERretrieval signal binds to COPI proteins and is required for transport from the Golgi to the ER. ${ }^{43)}$ Using a membrane-based lectin tetramer binding assay, we demonstrated that the N-terminal lectin domain preferentially recognizes the $\mathrm{B}$ isomer of $\mathrm{Man}_{8} \mathrm{GlcNAc}_{2}$ glycan (M8B), which is produced by trimming of an $\alpha 1,2$-linked mannose from the B-arm of the M9 high mannose-type glycan by $\alpha$ mannosidase I in the ER. ${ }^{44)}$ It is noteworthy to mention that the trimming of $\alpha 1,2$-linked mannose from the B-arm or C-arm of the M9 high mannosetype glycan critically distinguishes the two pathways by which glycoproteins are transported to the Golgi or to the ERAD, respectively.

ERGIC-53 has been identified as responsible for the autosomal recessive bleeding disorder called combined deficiency of coagulation factors $\mathrm{V}$ and VIII (F5F8D). The levels of factors V and VIII in the plasma of $\mathrm{F} 5 \mathrm{~F} 8 \mathrm{D}$ patients are $5-30 \%$ of those in healthy individuals, and $70 \%$ of $\mathrm{F} 5 \mathrm{~F} 8 \mathrm{D}$ patients have mutations in the gene encoding ERGIC-53. ${ }^{45)}$ F5F8D in the remaining $30 \%$ of patients is due to mutations in the gene that encodes MCFD2. ${ }^{46)}$,4) Using surface plasmon resonance and sedimentation equilibrium analytical ultracentrifugation, we showed that these two proteins form a stable complex in the presence of calcium concentrations $>0.2 \mathrm{mM}$, and further showed that MCFD2 binding to ERGIC-53 enhances the sugar-binding ability of ERGIC-53. ${ }^{44)}$ Furthermore, MCFD2 mutants in F5F8D patients exhibited decreased binding to ERGIC-53, resulting in a decrease in its sugar-binding activity. This supports the conclusion that the interaction between MCFD2 and ERGIC-53 is crucial for the secretion of coagulation factors $\mathrm{V}$ and VIII into the blood. Factors $\mathrm{V}$ and VIII have numerous $N$-glycosylation sites in their B domain, and following their secretion into the blood, they are processed to a B domaindeleted active form by thrombin. Kaufman's group produced recombinant B domain-deleted factor VIII in $\mathrm{CHO}$ cells; however, the B domain-deleted protein was not secreted sufficiently from the cells because the domain contains a region with more than six $N$ glycosylation sites, which are required for sufficient factor VIII secretion. ${ }^{48)}$ These results demonstrated that $N$-glycans on factor VIII are essential for its secretion by ERGIC-53. To investigate the role of $\mathrm{N}$-glycan-binding of ERGIC-53 in the transport of secretory glycoproteins, we searched for other ERGIC-53-transported cargo glycoproteins using a green fluorescent protein-based protein-fragmentcomplementation assay to screen a cDNA library obtained from HepG2 cells. Among several candidate ERGIC-53 interacting partners, we identified Mac-2 binding protein (Mac-2BP) as a novel cargo glycoprotein and showed that both $N$-glycan-binding of ERGIC-53 and the ERGIC-53-MCFD2 interaction are essential for the secretion of newly synthesized glycoproteins. ${ }^{49)}$ However, the significance of the ERGIC-53-MCFD2 interaction for glycoprotein transport via ERGIC-53 is controversial, based on 
the results of analyses using other model proteins, including cathepsin $\mathrm{C}$ and $\mathrm{Z}, \alpha 1$-antitrypsin and Mac-2BP. ${ }^{50)}$ The cooperative binding of $N$-glycan to the polypeptide portion appears to be the molecular basis for the selective cargo receptor function of ERGIC-53.

To understand the mechanism by which cargo proteins are released from ERGIC-53, we examined the interaction between ERGIC-53 and MCFD2 under several $\mathrm{pH}$ and calcium conditions using surface plasmon resonance. The calculated $K_{\mathrm{a}}$ values at $\mathrm{pH} 7.0,6.5$ and 6.0 were almost identical. By contrast, changes in the calcium concentration dramatically affected the interaction of the two molecules; $K_{\mathrm{a}}$ values at $0.05 \mathrm{mM}$ calcium were $100-$ 400 times less than at $1.0 \mathrm{mM}$ calcium. ${ }^{44)}$ The ERGIC is a complex membrane system that connects the ER and the Golgi, and has been proposed to be a specialized domain with a low calcium concentration that has evolved in higher organisms. ${ }^{51)}$ Under such conditions, cargo proteins bound to ERGIC-53/ MCFD2 complex via glycans may be released by the dissociation of MCFD2 from ERGIC-53. When cargo proteins are further transferred to the cisGolgi, association of ERGIC-53 and MCFD2 may occur again, restoring the sugar-binding activity of ERGIC-53. However, the glycans on cargo proteins will be processed into smaller $\mathrm{Man}_{5} \mathrm{GlcNAc}_{2}$ (M5) chains by Golgi $\alpha$-mannosidase I, resulting in the release of cargo proteins (Fig. 8). Thus, ERGIC-53/ MCFD2 then recycles to the ER without any associated cargo proteins. Zang et al. hypothesized that MCFD2 binds to coagulation factors V and VIII specifically, thus augmenting the transport of factors $\mathrm{V}$ and VIII into the cell. ${ }^{52)}$ However, a direct interaction between MCFD2 and factors V or VIII has not been demonstrated yet. Moreover, MCFD2 is constitutively expressed in all cells, suggesting that MCFD2 functions in the transport of multiple types of glycoproteins.

To achieve a better understanding of the mechanisms underlying the functional coordination of ERGIC-53 and MCFD2, X-ray crystallographic analysis of ERGIC-53 complexed with MCFD2 was performed. The lectin domain of human ERGIC-53 assumes a $\beta$-sandwich conformation consisting of two antiparallel $\beta$-sheets, two short $\beta$-strands, and three helices. The MCFD2-interacting site of ERGIC-53 is far from its carbohydrate-binding site, indicating that MCFD2 is not directly involved in sugar binding. ERGIC-53 complexed with MCFD2 showed no significant conformational alterations upon com-

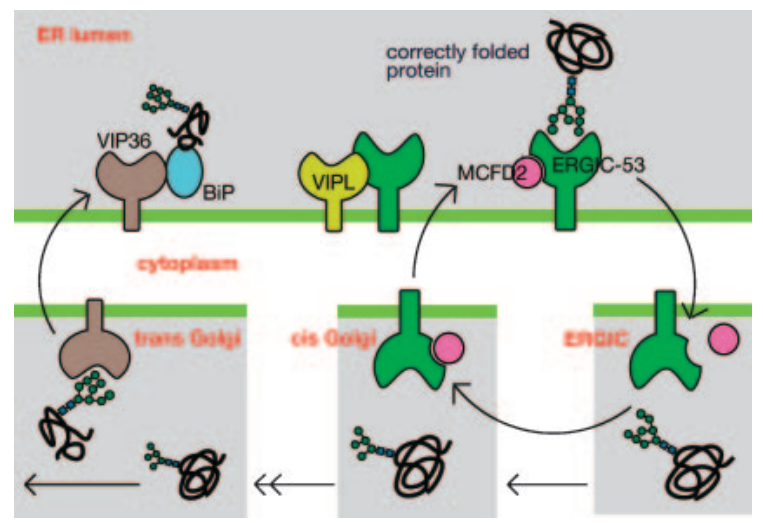

Fig. 8. Intracellular transport of cargo proteins via ERGIC-53/ MCFD2. When the protein has acquired its correctly folded structure in CNX cycle, $N$-glycan of the protein are processed from M9 to M8B by ER mannosidase I. ERGIC-53/MCFD2 preferentially binds to M8B glycan of cargo proteins in the presence of calcium. After cargo-ERGIC-53/MCFD2 is recruited to the ERGIC, cargo proteins bound to ERGIC-53/MCFD2 are released by the dissociation of MCFD2 from ERGIC-53 and then transported to the Golgi. In contrast, VIP36 transports misfolded glycoproteins from the Golgi to the ER and delivers them to $\mathrm{BiP}$

plex formation when compared with uncomplexed ERGIC-53. ${ }^{53)}$ Furthermore, analytical ultracentrifugation and NMR studies demonstrated that ERGIC53 and MCFD2 form a 1:1 complex, but not a multiunit complex in solution. ${ }^{44), 53)}$ Several missense mutations of the MCFD2 gene have been reported in F5F8D patients, and these mutations occur in the region of the gene encoding two EF-hand motifs. ${ }^{47}$ ) Half of these amino acid residues interact directly with ERGIC-53, while the other half are involved in calcium binding to maintain the structure of MCFD2. These data obtained from X-ray crystallographic analysis strongly suggest that MCFD2binding to ERGIC-53 is essential for the function of ERGIC-53; however, direct evidence showing that MCFD2 directly regulates the sugar binding of ERGIC-53 has not been demonstrated.

4.4. Retrograde transport of cargo proteins from the Golgi to the ER via VIP36. The cargo receptor vesicular integral membrane $36 \mathrm{kDa}$ protein (VIP36) also recycles between the ER and the Golgi. This protein is structurally similar to ERGIC-53 and consists of an L-type lectin domain, a stalk domain, a transmembrane domain, and a short cytoplasmic domain. However, it does not have a dilysine ERlocalization signal in its cytoplasmic tail and thus mainly localizes to the Golgi. This is supported by the observation that $N$-glycans of newly synthesized 
VIP36 rapidly become resistant to endo $\beta$ - $N$-acetylglucosaminidase $\mathrm{H}$ by processing of the $N$-glycans to complex-type $N$-glycans in the Golgi. VIP36 does not have cysteine residues and is present as a monomer under neutral $\mathrm{pH}$ conditions, whereas it forms oligomers at a $\mathrm{pH}$ of less than 6.0. The sugar-binding activity of VIP36 depends on $\mathrm{pH}$ and increases under low $\mathrm{pH}$ conditions. ${ }^{54)}$ The $\mathrm{pH}$ dependence of the sugar-binding ability of VIP36 is a unique property among the L-type lectin cargo receptors, ERGIC-53, VIP36 and VIPL. ${ }^{55)}$ The histidine residue (His178) of human ERGIC-53, which is located in the loop that mediates calcium binding, is responsible for a decrease in its sugar-binding activity at $\mathrm{pH}$ values below 7.0, which is the opposite effect seen for the sugar binding activity of VIP36. ${ }^{55)}$ The corresponding histidine residue, which is responsible for the decreased affinity for glycans under low $\mathrm{pH}$ conditions, is conserved in VIPL but not in VIP36. The $\mathrm{pH}$ values in lumen of the Golgi decrease gradually from the ER, through the cis- and medial-Golgi to the trans-Golgi. These conditions make it likely that VIP36 binds to ligands in the Golgi rather than in the ER. Using VIP36 tetramer binding in combination with membrane-based glycans and frontal affinity chromatography, we demonstrated that VIP36 binds to high mannose-type glycans, especially to higher molecular-weight ones, and specifically recognizes the Man $\alpha 1,2$ Man $\alpha 1,2$ Man sequence on the A-arm of high mannose-type glycans. ${ }^{54), 56)}$ Trimming of the $\alpha 1,2$-linked mannose residue from the Man $\alpha 1,2-$ Man $\alpha 1,2$ Man sequence on the A-arm or masking of the terminal $\alpha 1,2$-linked mannose by $\alpha 1,3$-linked glucose abrogates VIP36 binding.

Based on the $\mathrm{pH}$ dependence and sugar-binding specificity of VIP36, it has been proposed that VIP36 binds higher molecular-weight high mannose-type glycans in the Golgi, and retrogradely transports cargo proteins to the ER (Fig. 8). Using immunoprecipitation, tandem mass spectrometry, and immunoelectron microscopy, we demonstrated that VIP36 localized in the ER was constitutively associated with the molecular chaperone $\mathrm{BiP}$ in a sugar-independent manner, whereas VIP36 in the Golgi did not. ${ }^{57)}$ The interaction between VIP36 and $\mathrm{BiP}$ is dependent on divalent cations but not on ATP, whose mode of interaction is different from that observed between $\mathrm{BiP}$ and its chaperone substrates. Furthermore, increased expression of VIP36 was observed under conditions of ER stress. ${ }^{58)}$ Since trimming of $N$-glycans on misfolded proteins is not likely to occur immediately in the Golgi due to steric hindrance between Golgi-resident mannosidases and glycans on misfolded proteins, these observations may explain how VIP36 transports misfolded glycoproteins from the Golgi to the ER and delivers them effectively to BiP (Fig. 8).

VIPL is another L-type lectin cargo receptor localized in the ER. VIPL was first identified as a protein with high homology to VIP36 in silico, and revealed its expression in the ER. ${ }^{59)}$ Although VIPL also shows high structural similarity to VIP36, its characteristics are quite different from those of VIP36. VIP36 and VIPL have the same cytoplasmic ER-exit motif (KRFY) on their C-termini, but VIPL has an additional arginine at the fifth position from the C-terminus, which creates a diarginine ERlocalization motif (RKR). As a result, VIPL is a resident protein of the ER, unlike ERGIC-53 and VIP36. This is supported by the observation that $N$ glycans attached to VIPL were susceptible to endo $\beta$ $N$-acetylglucosaminidase $\mathrm{H}$ for more than $180 \mathrm{~min}$ after the translation was completed. We demonstrated that VIPL has sugar-binding activity in the presence of calcium and a sugar-binding specificity similar to that of VIP36. ${ }^{60)}$ In contrast, the optimum $\mathrm{pH}$ for the sugar-binding activity of VIPL is approximately 7.5, which is different from that of VIP36, whose optimum $\mathrm{pH}$ is more acidic. VIPL is able to bind to high mannose-type glycans with a Man $\alpha 1,2$ Man $\alpha 1,2$ Man sequence on the A-arm even in the neutral $\mathrm{pH}$ of the ER.

When cells were subjected to ER stress, there was a marked redistribution of ERGIC-53 from the ER to the Golgi, with a morphological change to a compact shape, whereas VIPL remained unchanged in its localization in the ER. Overexpression of ERGIC-53 also caused a similar redistribution of the protein to the Golgi, whereas the localization of ERGIC-53 to the ER was enhanced by co-expression with VIPL. ${ }^{58)}$ These results suggest that the distribution of ERGIC-53 is strictly regulated under conditions of ER stress and that VIPL may be involved in regulating the localization of ERGIC-53 in the cell. To clarify the influence of VIPL on ERGIC-53 distribution, we performed immunoprecipitation experiments using cell lysates expressing ERGIC-53 and/or VIPL. The results clearly showed that ERGIC-53 co-precipitated with VIPL, but not with VIP36, and that the ERGIC-53-VIPL interaction is mediated by the transmembrane or cytoplasmic domains of these cargo receptors (Fig. 8). ${ }^{58)}$ Under conditions of ER stress, several chaperones and intracellular lectins, which are involved in 
protein folding and ERAD, are upregulated through the unfolded protein response in the ER. When HeLa cells were subjected to ER stress for 14,24 and $42 \mathrm{~h}$, most of the chaperones, including CNX, calreticulin, BiP, EDEM1, FBG3, ERGIC-53, MCFD2, and VIP36, were increased by more than 5 -fold $24 \mathrm{~h}$ after the induction of ER stress. By contrast, VIPL was not upregulated, even after exposure to ER stress for more than $42 \mathrm{~h}$. This might explain why unfolded and misfolded glycoproteins were retained in the ER for further folding reactions. The Golgi-localization of ERGIC-53 could help to prevent misfolded or unfolded proteins in the ER from being transported to the Golgi.

4.5. Mannose 6-phosphate receptors for sorting of lysosomal enzymes. MPRs are another type of cargo receptors involved in the transport of lysosomal enzymes. These receptors have been extensively studied by several groups. ${ }^{61)}$ There are two kinds of MPRs, which are classified based on the type of divalent cation required for binding to ligands. Cation-independent receptors (CI-MPR) consist of 15-repeated mannose 6-phosphate receptor homology (MRH) domains in the luminal portion, followed by a transmembrane domain and a cytoplasmic domain. The MRH domain consists of approximately 150 amino acids, and each domain shows 16-38\% homology with the other domains. Among the repeated MRH domains, domains 3 and 9 have sugar-binding ability, and Asp103 in the manganese-binding loop is deleted in both domains, relieving their requirement for manganese ions for sugar-binding activity. ${ }^{62)}$ The second type of MPRs, cation-dependent MPR (CD-MPR), consists of an MRH domain, a transmembrane domain, and a cytoplasmic domain. It also has a tyrosine-based motif and an acidic cluster-dileucine motif in its cytoplasmic domain. Both CI-MPR and CD-MPR transport lysosomal enzymes with mannose 6-phosphate bearing $N$-glycans from the trans-Golgi network to the lysosome, and from the cell surface through the endosome to the lysosome. These two functions were confirmed by experiments showing that the transport of lysosomal enzymes in CI-MPRdeficient cells was recovered by transfection of CIMPR cDNA or treatment of the cell with anti-CIMPR and anti-CD-MPR antibodies. ${ }^{63), 64)}$ The result indicated that $60-70 \%$ of all lysosomal enzymes are transported to lysosome by CI-MPR, with the rest transported by CD-MPR.

All lysosomal enzymes have mannose 6-phosphate on the nonreducing termini of their high mannose-type glycans, synthesized by GlcNAc-1phosphotransferase and $\alpha$ - $N$-acetylglucosaminidase (uncovering enzyme) localized in the cis-Golgi and trans-Golgi network, respectively. ${ }^{65), 66)}$ GlcNAc-1phosphotransferase preferentially transfers GlcNAc1-phosphate to mannose on the C-arm of M8B high mannose-type glycans, and the trimming of $\alpha 1,2$ linked mannose from the B-arm by ER $\alpha$-mannosidase I enhances the transfer of GlcNAc-1-phosphate (Fig. 3B). ${ }^{66)}$ The uncovering enzyme is synthesized as a proenzyme and activated after removal of the N-terminal 24 amino acids by furin localized in the trans-Golgi network. ${ }^{66)}$ Thus, lysosomal enzymes are enriched in clathrin-coated vesicles in the transGolgi. ${ }^{67)}$ MPRs bind specifically to phosphorylated high mannose-type glycans with phosphorylated Man $\alpha 1,2$ Man sequences, but neither dephosphorylated high mannose-type glycans nor mannose 6phosphate binds to these receptors. If more than two mannose 6-phosphate residues are present on the same protein, its affinity for CI- and CD-MPRs is greatly increased, exhibiting $K_{a}$ values from $1.4 \times$ $10^{5} \mathrm{M}^{-1}$ to $5.0 \times 10^{8} \mathrm{M}^{-1}$ or $5.0 \times 10^{6} \mathrm{M}^{-1}$, respectively, ${ }^{68), 69)}$ which is explained by bivalent binding of the mannose 6-phosphates. The synthesis of mannose 6-phosphate on $N$-glycans has been extensively studied by Kornfeld's group, who demonstrated that the peptide portion of lysosomal enzymes is essential for the efficient transfer of GlcNAc-1-phosphate to high mannose-type glycans on lysosomal enzymes. ${ }^{70}$ Precise studies using lysosomal cathepsin D and non-lysosomal glycopepsinogen indicated that GlcNAc-1-phosphotransferase recognizes both several basic amino acids distributed widely across their molecular surface and high mannose-type glycans. ${ }^{71)}$

Mucolipidosis II and III are inherited lysosomal storage disorders caused by a deficiency of GlcNAc1-phosphotransferase. Mucolipidosis II is referred to as I-cell disease and the cells from patients with mucolipidosis II are rich in inclusion bodies that fail to be degraded by lysosomal enzymes. This defective enzymatic activity is caused by mutation of the gene encoding the GlcNAc-1-phosphotranferase $\alpha / \beta$ subunit. In addition, cells of patients with mucolipidosis IIIA (pseudo-Hurler polydystrophy) showed decreased activity of GlcNAc-1-phosphotransferase at $37^{\circ} \mathrm{C}$ and mucolipidosis IIIC cells exhibit defective recognition of lysosomal enzymes caused by mutation of $\gamma$-subunit. ${ }^{72)}$

CI- and CD-MPRs recycle between the Golgi, endosome, and cell surfaces. As they pass through 
these organelles, lysosomal enzymes from other proteins are sorted in the trans-Golgi network (TGN). In TGN, coat proteins, including AP-1 adaptin, form clathrin-coated pits and then transport vesicles to endosome. By contrast, AP-2 adaptin binds to CI-MPR on cell surfaces and forms endocytic vesicles in combination with other different coat proteins. Acidic cluster-dileucine motifs and tyrosinebased motifs in cytoplasmic domains of MPRs are involved in the binding of AP-1 and AP-2 adaptins, respectively. The binding of mannose 6-phosphate and MPRs is pH-dependent and occurs at a neutral $\mathrm{pH}$. Release is initiated at $\mathrm{pH}$ conditions $<6.0$. Thus, lysosomal enzymes transported via endocytic vesicles are released in acidic endosomes. ${ }^{62), 71)}$

4.6. E3 ubiquitin ligase recognizes $N$-glycan. Ubiquitinated proteins in the cytoplasm are destined unexceptionally for degradation by the $26 \mathrm{~S}$ proteasome. Ubiquitination of proteins is mediated by the ubiquitin-protein ligase complex, which consists of a ubiquitin-activating enzyme (E1), a ubiquitinconjugating enzyme (E2), and a ubiquitin-ligating enzyme (E3). Among these enzymes, E3 ligating enzymes recognize a variety of target proteins for ubiquitination. One of the ligating enzymes is the SCF complex, consisting of Skp1, Cullin1, Roc1 and F-box protein, which recruits substrates for ubiquitination and subsequent degradation by the proteasome. F-box protein has an N-terminal F-box domain that binds to Skp1, and a C-terminal substraterecognition domain. Based on their substrate-recognition domains, F-box proteins are classified into three subclasses, as follows: the Fbw family, which contains WD40 repeats; the Abl family, which contains leucine-rich repeats; and the Fbx family, members of which have a sugar-binding domain. Yoshida et al. demonstrated that Fbs1 (Fbx2) binds specifically to high mannose-type $N$-glycans and contributes to the ubiquitination of $N$-glycosylated proteins in the cytoplasm (Fig. 3B). ${ }^{73)} \mathrm{X}$-ray crystallographic analysis of the Fbs1 sugar-binding domain complexed with a sugar ligand demonstrated that the $\mathrm{Man}_{3} \mathrm{GlcNAc}_{2} \mathrm{~N}$-glycan core structure (Fig. 2) directly interacts with Fbs1. ${ }^{74}$ Core structure of $N$-glycans is the most common sequence among $N$-glycans, thus all $N$-glycosylated proteins are degraded without exception. It was demonstrated that Fbs2 (Fbx6b) also bound to $\mathrm{Man}_{3-9} \mathrm{GlcNAc}_{2}$ glycans and that the binding of $\mathrm{GlcNAc}_{2}$ was 3-4 orders of magnitude weaker than that of $\mathrm{Man}_{3-9} \mathrm{GlcNAc}_{2}{ }^{75)}$ Although the sugar-binding specificity of Fbs1 and Fbs2 is slightly different, Fbx proteins bind all glycoproteins that are translocated from the luminal side of the ER to the cytoplasm through recognition via OS-9 and/or XTP3-B (Fig. 7). These findings indicate that any misfolded glycoproteins in the cytoplasm are ubiquitinated and consequently degraded.

\section{Biological significance of the A, B, and C-arms of $\mathrm{N}$-glycan precursors}

As shown in Fig. 2, the biosynthetic pathway of $\mathrm{N}$-glycans is quite different from that of $O$-glycans. $N$-glycan precursors are assembled on the lipid dolichol, first on the cytoplasmic side of the ER and then on the lumenal side. Finally, synthesis of the $N$-glycan precursor is completed when the $\mathrm{Glc}_{3} \mathrm{Man}_{9} \mathrm{GlcNAc}_{2}$ (G3M9) structure is attached to the lipid dolichol. Transfer of the completed $\mathrm{N}$-glycan to newly synthesized polypeptides occurs on the lumenal side of the ER membrane by OST. The $N$ glycan precursor structure is conserved among eukaryotic organisms, including mammals, plants, insects, and fungi, and has three branches (A, B and $\mathrm{C}$-arms) on the core structure consisting of three mannose and two $N$-acetylglucosamine residues (Fig. 2, boxed in red). The B-arm and C-arm have Man $\alpha 1,2$ Man sequences and the A-arm has additional Glc $\alpha 1,2 \mathrm{Glc} \alpha 1,3 \mathrm{Glc}$ trisaccharides on Man $\alpha 1,2$ Man. When considering the glycan epitopes of intracellular lectins that are associated with quality control of glycoproteins, as described above (Fig. 3B), it is noteworthy to mention that the A, B, and $\mathrm{C}$-arms of $\mathrm{N}$-glycans are involved in folding, transport to the ER, and ERAD, respectively. The $N$-glycan precursor, G3M9, is not bound by any intracellular lectins. In contrast, removal of an outermost Glc residue from the A-arm induces folding of the nascent polypeptide associated with malectin. Further trimming of an additional Glc residue allows the nascent polypeptide to enter the CNX cycle and undergo correct folding. VIP36 preferentially recognizes deglucosylated A-arms and delivers $N$-glycan-unprocessed glycoproteins (which may be misfolded) from the Golgi to the ER, where the chaperone $\mathrm{BiP}$ enhances correct folding. VIPL, which also preferentially recognizes deglucosylated A-arms, may bring correctly folded proteins to ERGIC-53, the cargo receptor that transports the peptide from the ER though ERGIC to the Golgi. Following the trimming of an outermost Man residue from the B-arm of M9 glycans by ER-resident $\alpha$ mannosidase I, it can be bound by ERGIC-53 and initiate transport of the glycoprotein to the Golgi. 


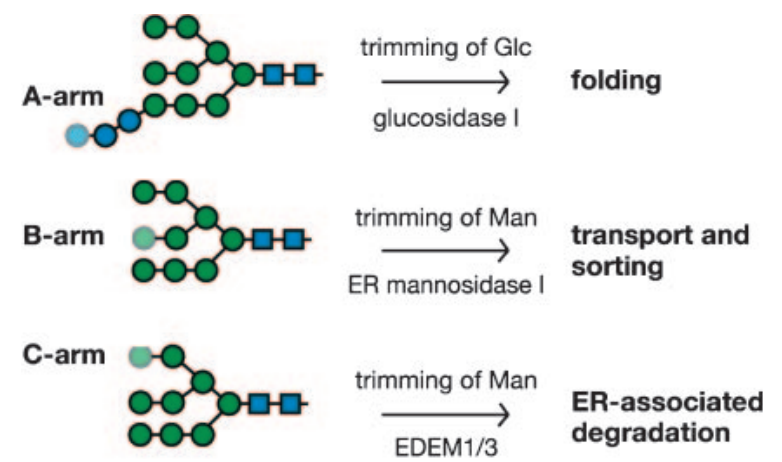

Fig. 9. Biological significance of the A, B, and C-arms of $N$ glycan. The A, B, and $\mathrm{C}$-arms of $N$-glycan are involved in folding, transport and sorting, and ERAD, respectively. Trimming of an outermost Glc or Man residue from each branch of $N$ glycan triggers the corresponding reaction with the assistance of intracellular lectins.

Trimming of an outermost Man residue from the B-arm also increases the phosphorylation of Man residues on $\mathrm{A}$ and $\mathrm{C}$-arms, which resulted in the transport to lysosome. When trimming of an outermost Man residue occurs on the $\mathrm{C}$-arm of high mannose-type glycans by EDEM1 or EDEM3, it binds OS-9 and XTP3-B. These soluble intracellular lectins will guide the protein to the ubiquitin ligase complex, and glycoproteins having such glycans will be degraded by the proteasome (Fig. 9). It is noteworthy that trimming of a terminal Man residue on the B or C-arm does not occur unless three Glc residues are removed from the A-arm, indicating that a folding step will occur at the beginning of the reaction, and transport to the Golgi or degradation will then occur during quality control of the glycoprotein.

Studies on intracellular lectins allow us to investigate the role of $N$-glycans in glycoprotein quality control in the ER. Meanwhile, the glycosylation and sugar processing that occur in the Golgi vary widely, both structurally and functionally, between cells, organs, tissues, and species. $N$-glycans transferred to newly synthesized proteins in the ER are then trimmed into smaller $\mathrm{Man}_{3} \mathrm{GlcNAc}_{2}$ chains and rebuilt into various complex-type glycans in the Golgi, resulting in the addition of diverse structures that enable the protein to play various roles outside the cell.

\section{Acknowledgements}

This work was supported in part by research grants from the JSPS, JST CREST, NEDO, and The Naito Foundation.

\section{References}

1) Varki, A., Cummings, R.D., Esko, J.D., Freeze, H.H., Stanley, P., Bertozzi, C.R., Hart, G.W. and Etzler, M.E. (1999) Essentials of glycobiology. Cold Spring Harbor Laboratory Press, New York.

2) Napetschnig, J. and Wu, H. (2013) Molecular basis of NF- $\kappa$ B signaling. Annu. Rev. Biophys. 42, 443468.

3) Sharon, N. and Li, H. (2003) Lectins. 2nd edn. Kluwer Academic Publishers, Boston.

4) Lee, R.T. and Lee, Y.C. (2000) Affinity enhancement by multivalent lectin-carbohydrate interaction. Glycoconj. J. 17, 543-551.

5) Knibbs, R.N., Takagaki, M., Blake, D.A. and Goldstein, I.J. (1988) The role of valence on the high-affinity binding of Griffonia simplicifolia isolectins to type A human erythrocytes. Biochemistry 37, 16952-16957.

6) Grakoui, A., Bromley, S.K., Sumen, C., Davis, M.M., Shaw, A.S., Allen, P.M. and Dustin, M.L. (1999) The immunological synapse: A molecular machine controlling T cell activation. Science 285, 221-227.

7) Yamamoto, K. and Kawasaki, N. (2010) Detection of weak-binding sugar activity using membranebased carbohydrates. Methods Enzymol. 478, 233-240.

8) Yamamoto, K. (2009) Intracellular lectins involved in folding and transport in the endoplasmic reticulum. Biol. Pharm. Bull. 32, 767-773.

9) Tateno, H., Nakamura-Tsuruta, S. and Hirabayashi, J. (2007) Frontal affinity chromatography: sugarprotein interactions. Nat. Protoc. 2, 2529-2537.

10) Varki, A. (1993) Biological roles of oligosaccharides: all of the theories are correct. Glycobiology 3, 97130.

11) Kornfeld, R. and Kornfeld, S. (1985) Assembly of asparagine-linked oligosaccharides. Annu. Rev. Biochem. 54, 631-664.

12) Schroder, M. and Kaufman, R.J. (2005) The mammalian unfolded protein response. Annu. Rev. Biochem. 74, 739-789.

13) Ellgard, L. and Helenius, A. (2003) Quality control in the endoplasmic reticulum. Nat. Rev. Mol. Cell Biol. 4, 181-191.

14) Kaji, H., Shikanai, T., Sasaki-Sawa, A., Wen, H., Fujita, M., Suzuki, Y., Sugahara, D., Sawaki, H., Yamauchi, Y., Shinkawa, T., Taoka, M., Takahashi, N., Isobe, T. and Narimatsu, H. (2012) Large-scale identification of $N$-glycosylated proteins of mouse tissues and construction of a glycoprotein database, GlycoProtDB. J. Proteome Res. 11, 4553-4566.

15) Jørgensen, F. and Kurland, C.G. (1990) Processivity errors of gene expression in Escherichia coli. J. Mol. Biol. 215, 511-521.

16) Chen, Y., Hu, D., Yabe, R., Tateno, H., Qin, S.Y., Matsumoto, N., Hirabayashi, J. and Yamamoto, K. (2011) Role of malectin in $\mathrm{Glc}_{2} \mathrm{Man}_{9} \mathrm{GlcNAc}_{2}-$ dependent quality control of $\alpha 1$-antitrypsin. Mol. Biol. Cell 22, 3559-3570.

17) Qin, S.Y., Hu, D., Matsumoto, K., Takeda, K., 
Matsumoto, N., Yamaguchi, Y. and Yamamoto, K. (2012) Malectin forms a complex with ribophorin I for enhanced association with misfolded glycoproteins. J. Biol. Chem. 287, 38080-38089.

18) Galli, C., Bernasconi, R., Soldà, T., Calanca, V. and Molinari, M. (2011) Malectin participates in a backup glycoprotein quality control pathway in the mammalian ER. PLoS ONE 6, e16304.

19) Kelleher, D.J., Kreibich, G. and Gilmore, R. (1992) Oligosaccharyltransferase activity is associated with a protein complex composed of ribophorin-I and ribophorin-II and a 48kd protein. Cell 69, 5565.

20) Wilson, C.M. and High, S. (2007) Ribophorin I acts as a substrate-specific facilitator of N-glycosylation. J. Cell Sci. 120, 648-657.

21) Wilson, C.M., Roebuck, Q. and High, S. (2008) Ribophorin I regulates substrate delivery to the oligosaccharyltransferase core. Proc. Natl. Acad. Sci. U.S.A. 105, 9534-9539.

22) Kleizen, B. and Braakman, I. (2004) Protein folding and quality control in the endoplasmic reticulum. Curr. Opin. Cell Biol. 16, 343-349.

23) Hammond, C., Braakman, I. and Helenius, A. (1994) Role of $N$-linked oligosaccharide recognition, glucose trimming, and calnexin in glycoprotein folding and quality control. Proc. Natl. Acad. Sci. U.S.A. 91, 913-917.

24) Zapun, A., Darby, N.J., Tessier, D.C., Michalak, M., Bergeron, J.J.M. and Thomas, D.Y. (1998) Enhanced catalysis of ribonuclease B folding by the interaction of calnexin or calreticulin with ERp57. J. Biol. Chem. 273, 6009-6012.

25) Sousa, M.C., Ferrero-Garcia, M.A. and Parodi, A.J. (1992) Recognition of the oligosaccharide and protein moieties of glycoproteins by the UDPGlc:glycoprotein glucosyltrtansferase. Biochemistry $31,97-105$.

26) Munro, S. (2001) The MRH domain suggests a shared ancestry for the mannose 6-phosphate receptors and other $\mathrm{N}$-glycan-recognizing proteins. Curr. Biol. 11, R499-R501.

27) Hu, D., Kamiya, Y., Totani, K., Kamiya, D., Kawasaki, N., Yamaguchi, D., Matsuo, I., Matsumoto, N., Ito, Y., Kato, K. and Yamamoto, K. (2009) Sugar-binding activity of the MRH domain in the ER $\alpha$-glucosidase II $\beta$ subunit is important for efficient glucose trimming. Glycobiology 19, 1127-1135.

28) Stigliano, I.D., Caramelo, J.J., Labriola, C.A., Parodi, A.J. and D'Alessio, C. (2009) Glucosidase II $\beta$ subunit modulates $N$-glycan trimming in fission yeasts and mammals. Mol. Biol. Cell 20, 3974-3984.

29) Deprez, P., Gautschi, M. and Helenius, A. (2005) More than one glycan is needed for ER glucosidase II to allow entry of glycoproteins into the calnexin/ calreticulin cycle. Mol. Cell 19, 183-195.

30) McCracken, A.A. and Brodsky, J.L. (1996) Assembly of ER-associated protein degradation in vitro: Dependence on cytosol, calnexin, and ATP. J. Cell Biol. 132, 291-298.
31) Carvalho, P., Goder, V. and Rapoport, T.A. (2006) Distinct ubiquitin-ligase complexes define convergent pathways for the degradation of ER proteins. Cell 126, 361-373.

32) Denic, V., Quan, E.M. and Weissman, J.S. (2006) A luminal surveillance complex that selects misfolded glycoproteins for ER-associated degradation. Cell 126, 349-359.

33) Gauss, R., Jarosch, E., Sommer, T. and Hirsch, C. (2006) A complex of Yos9p and the HRD ligase integrates endoplasmic reticulum quality control into the degradation machinery. Nat. Cell Biol. 8, 849-854.

34) Mikami, K., Yamaguchi, D., Tateno, H., Hu, D., Qin, S.Y., Kawasaki, N., Yamada, M., Matsumoto, N., Hirabayashi, J., Ito, Y. and Yamamoto, K. (2010) The sugar-binding ability of human OS-9 and its involvement in ER-associated degradation. Glycobiology 20, 310-321.

35) Yamaguchi, D., Hu, D., Matsumoto, N. and Yamamoto, K. (2010) Human XTP3-B binds to $\alpha 1$-antitrypsin variant null Hong Kong via the C-terminal MRH domain in a glycan-dependent manner. Glycobiology 20, 348-355.

36) Fujimori, T., Kamiya, Y., Nagata, K., Kato, K. and Hosokawa, N. (2013) Endoplasmic reticulum lectin XTP3-B inhibits endoplasmic reticulum-associated degradation of a misfolded $\alpha 1$-antitrypsin variant. FEBS J. 280, 1563-1575.

37) Satoh, T., Chen, Y., Hu, D., Hanashima, S., Yamamoto, K. and Yamaguchi, Y. (2010) Structural basis for oligosaccharide recognition of misfolded glycoproteins by OS-9 in ER-associated degradation. Mol. Cell 40, 905-916.

38) Song, X., Lasanajak, Y., Olson, L.J., Boonen, M., Dahms, N.M., Kornfeld, S., Cummings, R.D. and Smith, D.F. (2009) Glycan microarray analysis of P-type lectins reveals distinct phosphomannose glycan recognition. J. Biol. Chem. 284, 3520135214 .

39) Hosokawa, N., Tremblay, L.O., Sleno, B., Kamiya, Y., Wada, I., Nagata, K., Kato, K. and Herscovics, A. (2010) EDEM1 accelerates the trimming of $\alpha 1,2$-linked mannose on the $\mathrm{C}$ branch of $\mathrm{N}$-glycans. Glycobiology 20, 567-575.

40) Hosokawa, N., Wada, I., Hasegawa, K., Yorihuzi, T., Tremblay, L.O., Herscovics, A. and Nagata, K. (2001) A novel ER $\alpha$-mannosidase-like protein accelerates ER-associated degradation. EMBO Rep. 2, 415-422.

41) Gauss, R., Kanehara, K., Carvalho, P., Ng, D.T.W. and Aebi, M. (2011) A complex of Pdilp and the mannosidase Htm1p initiates clearance of unfolded glycoproteins from the endoplasmic reticulum. Mol. Cell 42, 782-793.

42) Schekman, R. and Orci, L. (1996) Coat proteins and vesicle budding. Science 271, 1526-1533.

43) Kappeler, F., Klopfenstein, D.R.C., Foguet, M., Paccaud, J.P. and Hauri, H.P. (1997) The recycling of ERGIC-53 in the early secretory pathway. J. Biol. Chem. 272, 31801-31808.

44) Kawasaki, N., Ichikawa, Y., Matsuo, I., Totani, K., 
Matsumoto, N., Ito, Y. and Yamamoto, K. (2008) The sugar-binding ability of ERGIC-53 is enhanced by its interaction with MCFD2. Blood 111, 1972-1979.

45) Nichols, W.C., Seligsohn, U., Zivelin, A., Terry, V.H., Hertel, C.E., Wheatley, M.A., Moussalli, M.J., Hauri, H.P., Ciavarella, N., Kaufman, R.J. and Ginsburg, D. (1998) Mutations in the ERGolgi intermediate compartment protein ERGIC53 cause combined deficiency of coagulation factors V and VIII. Cell 93, 61-70.

46) Zhang, B., Cunningham, M.A., Nichols, W.C., Bernat, J.A., Seligsohn, U., Pipe, S.W., McVey, J.H., Schulte-Overberg, U., Bosch, N.B., RuizSaez, A., White, G.C., Tuddenham, E., Kaufman, R.J. and Ginsburg, D. (2003) Bleeding due to disruption of a cargo-specific ER-to-Golgi transport complex. Nat. Genet. 34, 220-225.

47) Nyfeler, B., Kamiya, Y., Boehlen, F., Yamamoto, K., Kato, K., Moerloose, P., Hauri, H.P. and NeermanArbez, M. (2008) Deletion of three residues from the C-terminus of MCFD2 affects binding to ERGIC-53 and causes combined factor $\mathrm{V}$ and factor VIII deficiency. Blood 111, 1299-1301.

48) Miao, H.Z., Sirachainan, N., Palmer, L., Kucab, P., Cunningham, M.A., Kaufman, R.J. and Pipe, S.W. (2004) Bioengineering of coagulation factor VIII for improved secretion. Blood 103, 3412-3419.

49) Chen, Y., Hojo, S., Matsumoto, N. and Yamamoto, K. (2013) Regulation of Mac-2BP secretion is mediated by its N-glycan binding to ERGIC-53. Glycobiology 23, 904-916.

50) Appenzeller-Herzog, C., Nyfeler, B., Burkhard, P. Santamaria, I., Lopez-Otin, C. and Hauri, H.P. (2005) Carbohydrate- and conformation-dependent cargo capture for ER-exit. Mol. Biol. Cell 16, $1258-1267$.

51) Pezzati, R., Bossi, M., Podini, P., Meldolesi, J. and Grohovaz, F. (1997) High-resolution calcium mapping of the endoplasmic reticulum-Golgi-exocytic membrane system. Electron energy loss imaging analysis of quick frozen-freeze dried PC12 cells. Mol. Biol. Cell 8, 1501-1512.

52) Zheng, C., Liu, H.H., Yuan, S., Zhou, J. and Zhang, B. (2010) Molecular basis of LMAN1 in coordinating LMAN1-MCFD2 cargo receptor formation and ER-to-Golgi transport of FV/FVIII. Blood 116, 5698-5706.

53) Nishino, M., Kamiya, Y., Mizushima, T., Wakatsuki, S., Sasakawa, H., Yamamoto, K., Uchiyama, S., Noda, M., McKey, A.R., Fukui, K., Hauri, H.P. and Kato, K. (2010) Structural basis for the cooperative interplay between the two causative gene products of combined factor $\mathrm{V}$ and factor VIII deficiency. Proc. Natl. Acad. Sci. U.S.A. 107, 4034-4039.

54) Kamiya, Y., Yamaguchi, Y., Takahashi, N., Arata, Y., Kasai, K., Ihara, Y., Matsuo, I., Ito, Y., Yamamoto, K. and Kato, K. (2005) Sugar-binding properties of VIP36, an intracellular animal lectin operating as a cargo receptor. J. Biol. Chem. 280, $37178-37182$.
55) Kamiya, Y., Kamiya, D., Yamamoto, K., Nyfeler, B., Hauri, H.P. and Kato, K. (2008) Molecular basis of sugar recognition by the human L-type lectins ERGIC-53, VIPL and VIP36. J. Biol. Chem. 283, $1857-1861$.

56) Kawasaki, N., Matsuo, I., Totani, K., Nawa, D., Suzuki, N., Yamaguchi, D., Matsumoto, N., Ito, Y. and Yamamoto, K. (2007) Detection of weak sugar binding activity of VIP36 using VIP36-streptavidin complex and membrane-based sugar chains. J. Biochem. 141, 221-229.

57) Nawa, D., Shimada, O., Kawasaki, N., Matsumoto, N. and Yamamoto, K. (2007) Stable interaction of the cargo receptor VIP36 with molecular chaperone BiP. Glycobiology 17, 913-921.

58) Qin, S.Y., Kawasaki, N., Hu, D., Tozawa, H., Matsumoto, N. and Yamamoto, K. (2012) Subcellular localization of ERGIC-53 under endoplasmic reticulum-stress condition. Glycobiology 22, $1709-1720$.

59) Nufer, O., Mitrovic, S. and Hauri, H.P. (2003) Profile-based data base scanning for animal L-type lectins and characterization of VIPL, a novel VIP36-like endoplasmic reticulum protein. J. Biol. Chem. 278, 15886-15896.

60) Yamaguchi, D., Kawasaki, N., Matsuo, I., Totani, K., Tozawa, H., Matsumoto, N., Ito, Y. and Yamamoto, K. (2007) VIPL has sugar-binding activity specific for high-mannose-type $N$-glycans, and glucosylation of the $\alpha 1,2$-mannotriosyl branch blocks its binding. Glycobiology 17, 1061-1069.

61) Kornfeld, S. (1992) Structure and function of the mannose 6-phosphate/insulin-like growth factor II receptors. Annu. Rev. Biochem. 61, 307-330.

62) Hancook, M.K., Yammani, R.D. and Dahms, N.M. (2002) Localization of the carbohydrate recognition sites of the insulin-like growth factor II/ mannose 6-phosphate receptor to domains 3 and 9 of the extracytoplasmic region. J. Biol. Chem. 277, 47205-47212.

63) Kyle, J.W., Nolan, C.M., Oshima, A. and Sly, W.S. (1988) Expression of human cation-independent mannose 6-phosphate receptor $\mathrm{cDNA}$ in receptornegative mouse P388D1 cells following gene transfer. J. Biol. Chem. 263, 16230-16235.

64) Stein, M., Zijderhand-Bleekemolen, J.E., Geuze, H., Hasilik, A. and von Figura, K. (1987) Mr 46000 mannose 6-phosphate specific receptor: its role in targeting of lysosomal enzymes. EMBO J. 6, 26772681.

65) Encarnação, M., Kollmann, K., Trusch, M., Braulke, T. and Pohl, S. (2011) Post-translational modifications of the $\gamma$-subunit affect intracellular trafficking and complex assembly of GlcNAc-1-phosphotransferase. J. Biol. Chem. 286, 5311-5318.

66) Do, H., Lee, W.S., Ghosh, P., Hollowell, T., Canfield, W. and Kornfeld, S. (2002) Human mannose 6phosphate-uncovering enzyme is synthesized as a proenzyme that is activated by the endoprotease furin. J. Biol. Chem. 277, 29737-29744.

67) Do, H., Lee, W.S., Ghosh, P., Hollowell, T., Canfield, W. and Kornfeld, S. (2002) Human mannose 6- 
phosphoate-uncovering enzyme is synthesized as a proenzyme that is activated by the endoprotease furin. J. Biol. Chem. 277, 29737-29744.

68) Tong, P.Y., Gregory, W. and Kornfeld, S. (1989) Ligand interactions of the cation-independent mannose 6-phosphate receptor. J. Biol. Chem. 264, 7962-7969.

69) Tong, P.Y. and Kornfeld, S. (1989) Ligand interactions of the cation-dependent mannose 6-phosphate receptor. J. Biol. Chem. 264, 7970-7975.

70) Dustin, M.L., Baranski, T.J., Sampath, D. and Kornfeld, S. (1995) A novel mutagenesis strategy identifies distantly spaced amino acid sequences that are required for the phosphorylation of both the oligosaccharides of procathepsin $\mathrm{D}$ by $\mathrm{N}$ acetylglucosamine 1-phosphotransferase. J. Biol. Chem. 270, 170-179.

71) Kornfeld, S. and Mellman, I. (1989) The biogenesis of lysosomes. Annu. Rev. Cell Biol. 5, 483-525.

72) Little, L.E., Mueller, O.T., Honey, N.K., Shows, T.B. and Miller, A.L. (1986) Heterogeneity of $\mathrm{N}$ - acetylglucosamine 1-phosphotransferase within mucolipidosis III. J. Biol. Chem. 261, 733-738.

73) Yoshida, Y., Chiba, T., Tokunaga, F., Kawasaki, H., Iwai, K., Suzuki, T., Ito, Y., Matsuoka, K., Yoshida, M., Tanaka, K. and Tai, T. (2002) E3 ubiquitin-ligase that recognizes sugar chains. Nature 418, 438-442.

74) Mizushima, T., Yoshida, Y., Kumanomidou, T., Hasegawa, Y., Suzuki, A., Yamane, T. and Tanaka, K. (2007) Structural basis for the selection of glycosylated substrates by SCF Fbs1 ubiquitin ligase. Proc. Natl. Acad. Sci. U.S.A. 104, $5777-5781$

75) Yoshida, Y., Tokunaga, F., Chiba, T., Iwai, K., Tanaka, K. and Tai, T. (2003) Fbs2 is a new member of the E3 ubiquitin ligase family that recognizes sugar chains. J. Biol. Chem. 278, $43877-43884$.

(Received Nov. 8, 2013; accepted Dec. 6, 2013)

\section{Profile}

Kazuo Yamamoto was born in 1957, graduated from the Faculty of Pharmaceutical Sciences, The University of Tokyo in 1979. In 1984, he received a Ph.D. degree at the Department of Pharmaceutical Sciences, The University of Tokyo (Supervisor, Prof. Toshiaki Osawa). In the same year he moved to Tokyo Metropolitan Institute of Medical Sciences as a Research fellow and worked on the receptors on human platelets. In 1986, he returned to the Department of Pharmaceutical Sciences, The University of Tokyo as an Assistant Professor. In this period he interested in the relationship between the structures and sugar-binding specificities of lectins, and determined small carbohydratebinding peptides of several leguminous lectins, which were part of the conserved metalbinding loop. In 1997 he was appointed to an Associated Professor in the same group,

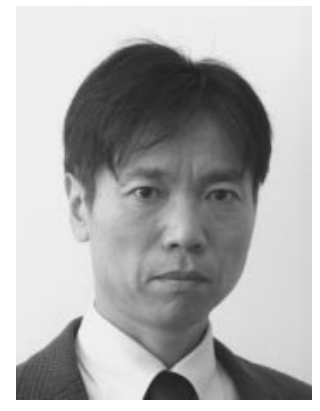
and in 1999 he moved to the Department of Integrated Biosciences, Graduate School of Frontier Sciences, The University of Tokyo as a Professor to start his studies on the functions of intracellular lectins and lectin-like receptors expressed in mammalian cells. 